\title{
UNA PANORÁMICA SOBRE PATENTES, VACUNAS Y COVID-19
}

\author{
AN OVERVIEW OF PATENTS, VACCINES AND COVID-19
}

UMA VISÃO GERAL DAS PATENTES, VACINAS E COVID-19

\author{
Benedicto Acosta \\ (Universidad de Salamanca) \\ bneacosta@usal.es \\ Mario A. Monzón Naranjo \\ (Universidad Pompeu Fabra) \\ marioantonio.monzon@alum.upf.edu
}

Recibido: 23/11/2021

Aprobado: 18/01/2022

\begin{abstract}
RESUMEN
El objetivo de este artículo es presentar una panorámica actualizada de la cuestión en torno a patentes y tratamientos de la COVID-19 y, especialmente, de las vacunas. Primeramente, ofrecemos una aclaración conceptual de conceptos como patente, vacuna o coronavirus. En segundo lugar, explicamos por dónde ha discurrido el debate entre propiedad intelectual y salud y abrimos paso para, en último lugar, reseñar los intentos de solución a los problemas abiertos y los desafíos que creemos que quedan por atender, con el objeto último de que todo ello sirva a las personas no expertas a orientarse mejor en esta intersección entre vacunas, COVID-19 y patentes.
\end{abstract}

Palabras clave: patentes. coronavirus. desafíos. tratamiento.

\section{ABSTRACT}

The aim of this article is to present an updated overview of the issue of patents and treatments for COVID-19 and, in particular, vaccines. First, we offer a conceptual clarification of concepts such as patent, vaccine, or coronavirus. Secondly, we explain where the debate between intellectual property and health has taken place and, finally, we outline the attempts to solve the open problems and the challenges that we believe remain to be met, with the aim of helping non-experts to find their way around this intersection between vaccines, COVID19 , and patents.

Keywords: patents. coronavirus. challenges. treatments.

\section{RESUMO}

O objetivo deste artigo é apresentar uma visão atualizada da questão das patentes e tratamentos para a COVID-19 e, em particular, das vacinas. Primeiro, oferecemos um esclarecimento conceitual de conceitos como patente, vacina ou coronavírus. Em segundo lugar, explicamos onde ocorreu o debate entre propriedade intelectual e saúde e, finalmente, 
esboçamos as tentativas de resolver os problemas abertos e os desafios que acreditamos ainda estarem por enfrentar, com o objetivo final de ajudar os não especialistas a encontrar seu caminho em torno desta interseção entre vacinas, COVID-19 e patentes.

Palavras-chave: patentes. coronavírus. desafios. tratamentos.

\section{Introducción}

El objetivo de este artículo es ofrecer una panorámica sobre el momento exacto en el que se encuentra el debate sobre las patentes de vacunas contra el COVID-19, así como presentar de una manera crítica desafíos abiertos para el debate ético y de gestión pública. Está dirigido a periodistas, informadores, divulgadores científicos, e incluso sanitarios $\mathrm{y}$, en general, personas que, sin ser expertas, quieran elaborarse un juicio informado sobre el tema.

Creemos que tal objetivo es necesario ante la falta de información generalizada -e incluso la mala información- que persiste en una gran parte de los relatos en medios de comunicación, que es la fuente de información más habitual de los que no son expertos en el asunto. Hemos detectado mucha confusión sobre conceptos importantes en el debate sobre patentes, vacunas y COVID-19. Por ejemplo, respecto a la posición del Parlamento Europeo, no solo de relevancia comunitaria sino muy influyente en el resto del planeta, se ha dicho que pretenden "suspender" las patentes (Domínguez, 2021), "liberarlas" (Martínez, 2021), "eliminarlas" (Mateos, 2021), un "levantamiento" (Sánchez, 2021), incluso que se ha llegado a pedir la "supresión" (Médicos Sin Fronteras, 2021). Todas estas ambigüedades y problemas se han repetido incluso en algunos artículos más académicos que, quizá por estar desactualizados, creemos que tampoco presentan adecuadamente la cuestión. Algunos de estos puntos los comentaremos a lo largo del escrito.

Por todo esto es necesario seguir incidiendo en la cuestión de las patentes, las vacunas y la COVID-19, pensando especialmente en todos aquellos que, quizá por su condición profesional, no lidien con los debates sobre la patentabilidad de las invenciones farmacéuticas y, sin embargo, se vean aludidos como filósofos, sanitarios o ciudadanos. Y esta empresa cobra aún más importancia teniendo en cuenta la falta artículos destinados al público hispanohablante en revistas académicas, algo que puede comprobarse fácilmente a través de una búsqueda en bases de datos bibliográficas.

Así pues, el artículo seguirá la siguiente estructura: primeramente, ofrecemos una aclaración conceptual de conceptos como patente, vacuna o coronavirus. En segundo lugar, explicamos por dónde ha discurrido el debate entre propiedad intelectual y salud y abrimos paso para, en último lugar, reseñar los intentos de solución a los problemas abiertos y los desafíos que creemos que quedan por atender.

\section{Marco conceptual}

Antes de nada, parece oportuno definir ciertos conceptos que están indisociablemente ligados a la discusión social y política sobre las patentes de las vacunas de la COVID-19. En primer lugar, parece importante responder a la pregunta de qué es y qué protege una patente, puesto que las patentes son documentos complejos, a la vez jurídicos y altamente técnicos, con una especificidad nacional muy marcada. En segundo lugar, trataremos de aclarar la cuestión de los tratamientos sobre la COVID-19, especialmente algunos puntos concernientes a la figura de la vacuna.

Las patentes son derechos que los inventores obtienen y que les permiten, por un período limitado de tiempo, prohibir a terceros la fabricación y comercialización de sus técnicas o artefactos. La idea clásica es que, a cambio, estos derechohabientes levantan del secreto industrial dicho invento y lo hacen público en los documentos de patentes, que se supone sirven para fomentar de nuevo otras innovaciones y, con ello, hacen posible el cambio y progreso tecnológico (Nordhaus, 1969; Denicolò, 2002). 
Los coronavirus $(\mathrm{CoV})$, por su parte, son una subfamilia de virus de ARN monocatenario, que producen patologías ya conocidas como el síndrome respiratorio agudo severo (SARS-CoV), el síndrome respiratorio de Oriente Medio (MERS-CoV) y el propio SARS-CoV-2 (que causa la COVID-19). Algo de vital importancia es que todos ellos presentan cuatro proteínas estructurales, entre la que destaca la proteína de la espícula $S$, que tiene un papel relevante en el desarrollo de la vacuna para el COVID-19, puesto que al ser una proteína común a los diferentes tipos de coronavirus los científicos ya la han empleado con la vacuna del SARS-CoV en 2003 (Dong, et al., 2020; Uddin, et al., 2020).

Las vacunas, y entre ellas las de la COVID-19, son productos biológicos que provocan la respuesta inmune a un antígeno específico derivado del patógeno causante de una enfermedad infecciosa. Estos suelen producirse mediante la manipulación de organismos vivos y/o de material genético (Aars, Clark, \& Schwalbe, 2021; Czochor \& Turchick, 2014). En tanto que productos farmacéuticos y biológicos, las vacunas pueden caer bajo la protección de las patentes, como de hecho lleva ocurriendo desde hace décadas. Pero si nos centramos en aspectos del desarrollo, la producción, las limitaciones reglamentarias y la estructura de las patentes, no debemos homologar el término "vacuna" y "fármaco" (Aars, et al., 2021). Mientras que los fármacos son químicamente idénticos y, por tanto, pueden ser copiados mediante ingeniería inversa por otros fabricantes para producir fármacos bioequivalentes, las vacunas requieren pasar por los mismos procedimientos y ensayos clínicos que el producto original, debido a la complejidad de su estructura y los procesos de fabricación (cultivos de células animales, bacterianas o levaduras...) (Aars, et al., 2021).

Así pues, en caso de una emergencia de salud pública es más fácil distribuir de forma rápida un fármaco que una vacuna, ya que en estas son patentables tanto los componentes como los procesos, el adyuvante e, incluso, los virus inactivos y la estructuras genéticas (M. Eccleston-Turner, 2017).

Es un hecho, sin embargo, que las vacunas contra la COVID-19 se han revelado como uno de los tratamientos más efectivos. El interés de este artículo está en resaltar precisamente las dificultades políticas y sociales que conciernen a la protección industrial de estas vacunas, precisamente por eso es oportuno hacer ciertas aclaraciones.

La primera tiene que ver con el hecho de que las patentes no protegen todo lo relativo a un tratamiento. Es habitual que las empresas farmacéuticas registren también marcas (el nombre comercial, por ejemplo), pero incluso que mantengan en el secreto industrial ciertos métodos de fabricación o el knowhow. La segunda puntualización es la inversa de esta: que existen más patentes importantes para el tratamiento de la COVID-19 que no son las de vacunas, como las de otros fármacos o tests de diagnóstico. Un estudio realizado por la OMPI/WIPO en 2012 clasificó las patentes relevantes para los tratamientos integrales de la gripe y otras enfermedades respiratorias causadas por virus. En él aparecen desde componentes de vacunas como los adyuvantes a procesos de producción como la purificación o la conservación mediante congelación de las vacunas, etc. (cf. WIPO 2012). Todo ello nos revela el número de protecciones que existen en torno a una vacuna clásica, y da una idea al mismo tiempo de cuál debe ser la red de patentes de las vacunas llamadas "de nueva generación".

Entrando propiamente en las vacunas contra la COVID-19, son dos las clasificaciones más habituales para diferenciar sus características: las vacunas "clásicas" (entre las que se encuentran vacunas vivas atenuadas y vacunas inactivadas) y las vacunas de nueva generación, como las vacunas de ácidos nucleicos (ADN o ARNm), las de vector (viral, bacteriana, etc.), las de proteína recombinante o las de células presentadores de antígenos (Dong, et al., 2020; Uddin, et al., 2020; van Riel \& de Wit, 2020; Wang, Peng, Xu, Cui, \& Williams, 2020) -véase Figura 1-.

Dentro de las vacunas tradicionales, nos encontramos en primer lugar con las vacunas inactivadas, que mediante métodos físicos o químicos provocan que el virus no pueda replicarse (Dong et al., 2020). Este tipo de vacunas puede producirse fácilmente a gran escala. No obstante, presentan el problema de requerir adyuvantes para estimular el sistema inmunológico y de necesitar múltiples dosis para establecer la memoria inmunitaria (van Riel \& de Wit, 2020; Wang et al., 2020). Por otra parte están las vacunas de virus vivos atenuados, que producen una respuesta inmunitaria fuerte y cuya memoria inmunológica 
puede durar años, pero en ocasiones no es conveniente aplicarlas a personas inmunodeprimidas (Dong et al., 2020; van Riel \& de Wit, 2020; Wang et al., 2020). En las vacunas clásicas, además de las comentadas previamente, existen limitaciones que son comunes, y que dificultan la producción rápida de vacunas en contextos de pandemia, como las pruebas de seguridad exhaustivas y el cultivo de grandes cantidades de virus en condiciones de nivel de bioseguridad 3 (BSL3) (van Riel \& de Wit, 2020).

Pero lo anterior es diferente en las vacunas llamadas "de nueva generación", puesto que al conocer la estructura y la inmunopatogénesis del patógeno, precisamente por ser homólogo del síndrome respiratorio agudo severo (SARS-CoV) y el síndrome respiratorio de Oriente Medio (MERS-CoV), hace a estas más fácilmente adaptables y acelera el desarrollo del producto (van Riel \& de Wit, 2020; Wang et al., 2020).

Entre estas últimas cabe destacar las de ácido nucleico, como las de ADN y las de ARNm. Estas se administran en células humanas con el objeto de transcribirse más adelante en proteínas virales principalmente la proteína S (Dong, et al., 2020). Desde el punto de vista industrial, la vacuna de ADN tiene como ventajas una excelente biocompatibilidad del ADN plasmídico, una vida larga útil y fabricación de bajo coste (van Riel \& de Wit, 2020). Las de ARNm tienen una capacidad de desarrollo rápido, alta potencia y una producción rentable (inmunidad protectora a dosis más bajas); sin embargo, las propiedades fisicoquímicas del ARNm pueden influir en el suministro y distribución.

En ambos casos existe una baja eficacia de transfección (esto es, la introducción de material genético externo en las células), por la cual necesitan, en el caso de la vacuna de ADN, electroporación para atravesar la membrana nuclear, o para las de ARNm, el uso de nanopartículas de lípidos (LNP de su acrónimo en inglés) (Dong, et al., 2020; van Riel \& de Wit, 2020; Wang, et al., 2020).

Pero, ¿cómo de hecho se distribuyen y administran a gran escala en el contexto de la pandemia? ¿A qué desafíos se enfrentan los países para favorecer un acceso equitativo a las vacunas y los tratamientos? O ¿cómo se protegen estas vacunas mediante propiedad intelectual? Trataremos de explicarlo en lo que sigue.

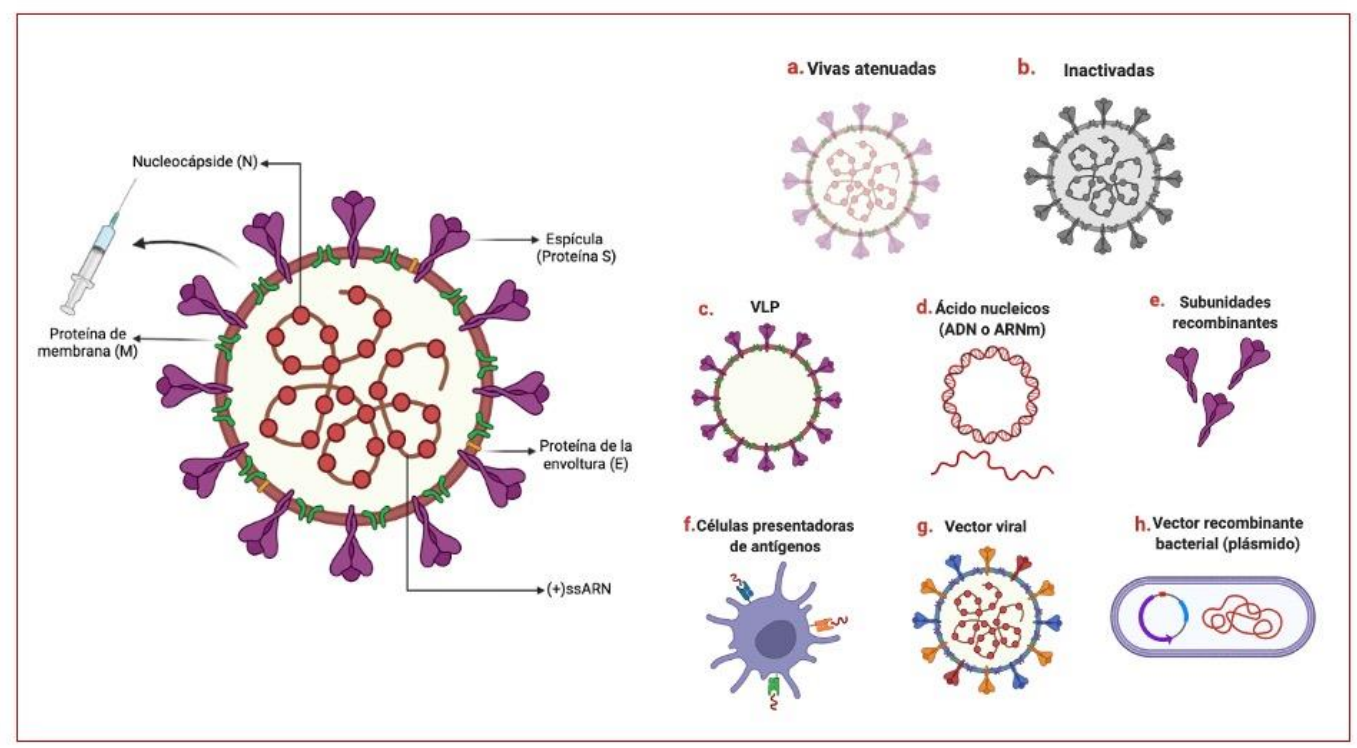

Figura 1. Representación esquemática de la estructura del SARS-CoV-2 y las vacunas clásicas y de próxima generación (Dong, et al., 2020; van Riel \& de Wit, 2020).

\section{Debates}


La polémica en torno a la licitud de las patentes en cuestiones de salud pública es mucho anterior a la enfermedad por el nuevo coronavirus. De hecho, es uno de los debates más clásicos en la ética de la propiedad industrial (cf. Dutfield 2003). Uno de los casos que suelen argüirse es que las patentes dificultan la investigación y la creación de nuevo conocimiento. Las patentes son para las empresas un tipo de estrategia y, como tal, pueden tener el objetivo de impedir que otros innoven antes incluso que la protección de las propias tecnologías (Biddle 2014). Esto está especialmente estudiado en el sector químico y farmacéutico, donde las solicitudes de patentes reivindican una protección muy amplia (Chandrasekharan, et al. 2015: 6369).

Entrando en este sector biosanitario, son muchos los autores que creen que las compañías farmacéuticas imponen unos precios abusivos a sus productos sirviéndose del monopolio que le confieren las patentes (Parthasarathy, 2017). El argumento es que, como no hay competencia que estimule la bajada de precios, el titular de un medicamento patentado puede poner el precio que desee, máxime si las personas enfermas que requieren ese tratamiento no tienen otra alternativa para sanar; llegado el caso, imponen esos precios a los Estados que financian dichos fármacos. Este tipo de razonamiento no se limita a la discusión académica, sino que tiene eco en los medios de comunicación y la opinión pública. Un caso muy sonado ha sido el de una patente, vigente en la UE, que reivindica el uso de productos que contienen sofosbuvir en el tratamiento contra la hepatitis $C$ crónica. La compañía farmacéutica que posee la licencia vendía esos productos a un precio que supera las decenas de miles de euros (cf. Henry, 2018).

Ahora bien, y dado su carácter controvertido, pensamos que hay ciertas cosas que aclarar brevemente en esta discusión, que desde luego tiene también influencia en el debate que nos ocupa, sobre patentes, COVID-19 y vacunas. Primeramente, el titular de una patente puede negar a terceros la explotación comercial del invento sobre el que posee tal derecho, pero eso no le permite ni siquiera comerciar con su invención, que muchas veces requiere de autorizaciones también muy difíciles, sobre todo en cuestiones de salud (autorizaciones de las agencias reguladoras del medicamento). En segundo lugar, la exclusividad de los derechohabientes sobre, por ejemplo, un principio activo novedoso, no siempre genera monopolios. Es muy posible que aparezcan en el mercado patentes de medicamentos que son químicamente diferentes pero que actúan sobre los síntomas de la misma enfermedad. Eso genera una competición no monopolística entre empresas que poseen patentes distintas, pero con fines terapéuticos afines (Cebrián y López, 2017). Por todo esto, creemos que es oportuno estudiar cada caso concreto, y no tanto elevarlo a una afrenta general contra las patentes. Sobre todo, teniendo en cuenta que las licencias obligatorias (una figura que permite a los gobiernos suspender la exclusividad de una patente) o las expropiaciones pueden ser un desincentivo a la innovación y algo que también afecta al retorno de inversión de farmacéuticas.

Ahora bien, no es menos cierto que el problema del precio de los tratamientos ha cobrado también sentido a la luz de la COVID-19. Un caso ha sido el del remdesivir, un medicamento recomendado para tratar casos graves de la enfermedad (Agencia Española del Medicamento, 2020). El tratamiento de cinco días con este fármaco se vendía a más de tres mil dólares en los Estados Unidos, por mucho que se comerciaba con él a un precio mucho más bajo en países en vías de desarrollo (Perehudoff, Hoen y Boulet, 2021: 2), algo que parece demostrar que el elevado coste no tenía como fin compensar los costes de la investigación, sino más bien obtener cuantiosos beneficios a costa de una situación excepcional desde el punto de vista político y moral. De hecho, Rusia aprobó una licencia obligatoria para este fármaco a finales de 2020 (The Pharma Letter, 2021).

Otro argumento que problematiza la patentabilidad de las invenciones sanitarias y que tiene mucha relevancia en el caso del nuevo coronavirus, es que existen medicamentos patentados por empresas cuya investigación ha sido en parte, o totalmente, financiada con dinero público. Esta situación se agrava cuando se trata de compañías que han empleado para su vacuna tecnologías previamente protegidas por patentes para las que en su momento se concedieron licencias, sin permitir ellos, a su vez, las investigaciones y el desarrollo en vacunas de mRNA (cf. Gaviria y Kilic, 2021). 
Ahora bien, también es cierto que muchas compañías farmacéuticas han hecho público sus compromisos de colaborar en la producción de vacunas y su intención de no lucrarse y, dado que la competencia crece entre las opciones a un tratamiento y que eso puede generar una situación no-monopolística, de competencia, parece una medida a considerar. El acuerdo AstraZeneca y Universidad de Oxford es uno de los más sonados, y por su carácter público-privado ha tenido relevancia para la situación anterior.

Así las cosas, existen en la bibliografía dos formas que se solicitan garantizar que la protección de la PI no impida el acceso a nuevos medicamentos, tanto para la COVID-19, como en el debate más amplio de las invenciones biotecnológicas: la primera, que los dueños de dicha patente ofrezcan precios asequibles y un suministro adecuado mediante licencias voluntarias; la segunda, las licencias obligatorias (cf. Perehudoff, Hoen y Boulet, 2021). Estos factores los discutiremos en mayor medida a lo largo del siguiente punto.

\section{Intentos de solución y desafíos}

Uno de los problemas a la hora de producir vacunas a gran escala es que implican a los derechos de propiedad intelectual y la limitación de conocimientos que estos pueden crear (McMahon, 2020). Tal como se comentó previamente, la producción de las vacunas lleva consigo una serie de especificidades que las hacen distintas al resto de fármacos (Aars, et al., 2021; M. Eccleston-Turner, 2017). Además, no solo serían necesarios los conocimientos de fabricación sino el acceso a la información secreta comercial o las líneas celulares empleadas (McMahon, 2020).

Una forma de obtener dicha información sería mediante la concesión de licencias obligatorias. Estas permiten a un tercero producir una tecnología patentada sin el permiso del titular de la patente. El artículo 31 del Acuerdo sobre los Aspectos de los Derechos de Propiedad Intelectual relacionados con el Comercio (ADPIC) (WTO, n.d.) permite a todos los Estados de la Organización Mundial del Comercio (OMC) emitir licencias obligatorias con sujeción a determinados criterios. El motivo por el que no se emplean estas licencias para producir las vacunas para COVID-19 a gran escala es que se deben obtener licencias obligatorias individuales para cada uno de los Estados y para cada una de las invenciones que se deseen (vacuna, adyuvantes, proceso, etc.), algo que ralentiza el procedimiento en el contexto de una pandemia (McMahon, 2020).

Por ello, se ha apreciado una opción nueva ante este dilema: las licencias voluntarias. Estas se plantean antes de emitir una licencia obligatoria ya que presentan condiciones más favorables de cara al titular de patentes. Una de las iniciativas más sonadas para promover las licencias voluntarias para COVID-19 ha sido Covid Technology Access Pool (C-TAP), de la Organización Mundial de la Salud (OMS) (McMahon, 2020; WHO, n.d.-b).

C-TAP tiene como objetivo acelerar el desarrollo de los productos necesarios para combatir la COVID19, al fomentar el intercambio de derechos de propiedad intelectual (McMahon, 2020; WHO, n.d.-a; WHO, n.d.-b). Este intercambio de información agiliza el desarrollo de los productos y evita duplicaciones en las investigaciones futuras (WHO, n.d.-a). No obstante, las expectativas depositadas en C-TAP no se han cumplido, debido mayoritariamente a la negativa de la industria farmacéutica a participar (Health Action International, n.d.). El periódico The Guardian es tajante al respecto: "no ha traído contribuciones en los ocho meses desde que se estableció" (The Guardian, n.d.).

Por su parte, la Organización Mundial de la Salud (OMS) en abril de 2020 junto con la Coalición para las Innovaciones en la Preparación ante Epidemias (CEPI), la alianza de vacunas (GAVI) y otros socios como UNICEF, propusieron el Acelerador del acceso a las herramientas contra la COVID-19 o ACT-A (cf. Asundi, O'Leary, \& Bhadelia, 2021; Mark Eccleston-Turner \& Upton, 2021; McAdams, et al., 2020; Peacocke, Heupink, Frønsdal, Dahl, \& Chola, 2021; van de Pas et al., 2021). ACT-A coordina la estrategia, la financiación y el desarrollo, entre otras, desde cuatro áreas o pilares (Mark EcclestonTurner \& Upton, 2021; van de Pas et al., 2021): el diagnóstico, la terapéutica, las vacunas (llamadas COVAX) y el fortalecimiento de los sistemas de salud (véase ). 
COVAX, a su vez, se enfoca en lograr tres objetivos: acelerar rápidamente el desarrollo de vacunas para COVID-19 proporcionando respaldo financiero a una variedad de candidatos prometedores, el uso de mecanismos de financiación de "incitación y disuasión" (push and pull) para estimular la inversión en riesgo en la capacidad de fabricación y asegurar el acceso equitativo a nivel mundial (Mark EcclestonTurner \& Upton, 2021). COVAX se marcó como meta asegurar al menos dos mil millones de dosis de la vacuna COVID-19 para finales de 2021, donde más del 60\% serían destinadas a 92 países de bajos ingresos (Forman, Shah, Jeurissen, Jit, \& Mossialos, 2021; McAdams, et al., 2020; Peacocke, et al., 2021). A fecha de octubre de 2021 "solo se han enviado 371,1 millones de dosis... queda por ver si esta meta se alcanzará en 2022" (Krishtel \& Hassan, 2021). Esto puede retrasarse además por el déficit de financiación de 3.7 mil millones de dólares que tiene COVAX en 2020 y que Estados Unidos ha prometido ayudar a corto plazo junto con los 23.7 mil millones solicitados para 2021(Asundi, et al.,2021; Mark Eccleston-Turner \& Upton, 2021).

De igual forma, se estipuló en un inicio que estas dosis provendrían de CEPI, asociación público-privado, y en un futuro se irían obteniendo vacunas de otras fuentes para asignarlas a todos los países participantes de forma igualitaria y en proporción al tamaño total de su población (McAdams, et al., 2020). Este último punto ha despertado críticas por no haberse distribuido las vacunas según la gravedad de la situación o la resistencia de los sistemas de salud afectados por aumentos repentinos de nuevos brotes (Asundi, et al., 2021). El hecho es que los países con un poder adquisitivo mayor han recibido más del $87 \%$ de las vacunas mientras que los países con ingresos medianos o bajos solo el 0,2\% (Peacocke, et al., 2021). Además, como muchos países priorizan en su vacunación nacional frente a la internacional la meta de COVAX se ha visto obstaculizada (van de Pas, et al., 2021).

Pero estas no son las únicas barreras que dificultan los objetivos planteados por COVAX para finales de 2021. No podemos obviar el hecho de que esta organización debe competir con los acuerdos bilaterales entre países de altos ingresos y empresas farmacéuticas (Asundi, et al., 2021). Según los datos recopilados por el Centro de Innovación Global de Duke, COVAX reservó solo 700000 dosis de vacunas frente a los 6000 millones de dosis que los países de altos ingresos firmaron a través de acuerdos bilaterales con empresas farmacéuticas (Usher, 2020). Aunque estos países han comenzado a hacer donaciones y tener un mayor compromiso de dosis con COVAX, hasta la fecha estas donaciones no se acercan a la escala de lo que se necesita. De hecho, China y Rusia, que no participaron en COVAX, están proporcionando dosis de vacunas desarrolladas a nivel nacional (Sputnik, Sinopharm y Sinovac) a través de acuerdos bilaterales (Asundi, et al., 2021). A lo que hay que sumar que algunos países han implementado incluso prohibiciones de exportación de vacunas, como el caso de Alemania con Italia (cf. Forman, et al., 2021).

Una dificultad común a la exportación mediante acuerdos nacionales o multilaterales es que se ha visto cómo los requisitos de datos, los procesos de concesión de licencias, los resultados de los estudios o el empleo de nuevas tecnologías pueden dificultar el transporte de las vacunas. COVAX no ha contratado para países más desfavorecidos el suministro de la vacuna Moderna, aprobada en EEUU y Canadá a finales de diciembre de 2020 y en la Unión Europea en enero de 2021, por la inestabilidad de utilizar la tecnología de ARNm que debe almacenarse a $-70^{\circ} \mathrm{C}$ (Mark Eccleston-Turner \& Upton, 2021). Además, como ya se ha apuntado en el anterior capítulo, "la negativa de Moderna a otorgar licencias de tecnología a otros fabricantes ha sido criticada, ya que se desarrolló principalmente con fondos públicos" (Krishtel \& Hassan, 2021). Este punto es crucial ya que CEPI, cofundador de COVAX, aportó grandes subvenciones a una cartera geográficamente diversa de vacunas candidatas (AstraZeneca, Novavax, Moderna y Pfizer) con el compromiso de suministrar vacunas a países en desarrollo, fijar precios asequibles y transferir tecnología. AstraZeneca/Oxford y Novavax asumieron esto en mejor medida que Moderna y Pfizer (Mark Eccleston-Turner \& Upton, 2021; Moon, Alonso Ruiz, \& Vieira, 2021).

Varios miembros del Senado y el Congreso de los Estados Unidos lo expresan así en una carta reciente: "A pesar de recibir enormes sumas de fondos públicos de los contribuyentes estadounidenses, Moderna ha rechazado las peticiones para compartir su tecnología, incluso las del Gobierno de los Estados Unidos" (Warren, 2021). 
A su vez, la confianza en la seguridad y eficacia en las vacunas está frenando el despliegue de la vacunación COVID-19: en Europa una gran cantidad de dosis de la vacuna AstraZeneca no se han usado debido a las discrepancias sociopolíticas en la inoculación a adultos mayores (Mark Eccleston-Turner \& Upton, 2021; Forman, et al., 2021). Y otro factor a tener en cuenta es el precio de las vacunas COVID19, ya que informes sugieren que pagan más los países con un poder adquisitivo menor y un índice de pobreza mayor; por tanto, existe una amplia variación de los precios entre los diferentes países: Arabia Saudita, Uganda y Sudáfrica están pagando más de \$ 5 por dosis por la vacuna Oxford/AstraZeneca, y la Comisión Europea paga \$ 3,50 por dosis(Forman, et al., 2021). Sin embargo, AstraZeneca y también Johnson\&Johnson se han comprometido a vender sus vacunas a precio de costo durante un período de tiempo (Forman, et al., 2021; Matheson Am \& Kirkinis, 2021).

Todos estos problemas plantean el hecho de cómo se puede incentivar a los gobiernos para que diseñen de forma coordinada un buen modelo de fabricación distributiva. Forman, R., Shah, S., Jeurissen: , Jit, M., \& Mossialos, E. (2021) plantean que las agencias internacionales subvencionen a países con una población reducida, pero con una gran capacidad de fabricación para que las distribuyan; de esto modo se podría obtener una mayor oferta en los países más necesitados. Además, las normas internacionales podrían garantizar un intercambio rápido de datos y resultados que permitirían obtener información de posibles variantes emergentes, como sucedió en la secuenciación genómica sobre el SARS-CoV-2 que científicos chinos difundieron mediante la plataforma de acceso público GISAID (Moon et al., 2021).

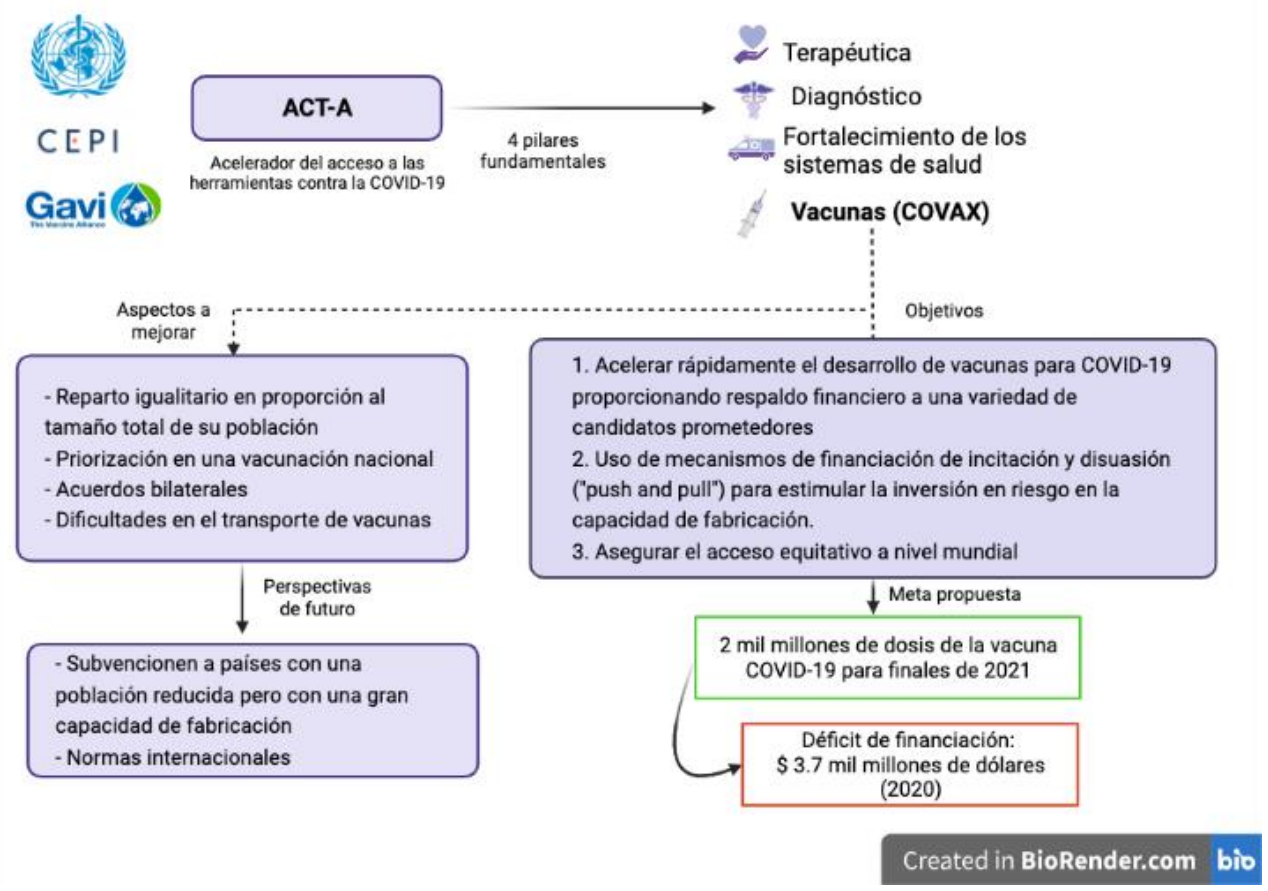

Figura 2. En esta tabla resumimos el estado de los acuerdos y los desafíos discutidos.

Sobre esta posibilidad de que algunos países distribuyan vacunas a los más necesitados, es oportuno hablar del caso de Europa. La mayoría de Las Constituciones de países europeos explicitan que la propiedad privada está subordinada al bien común y al interés general (por ejemplo, la Constitución Española en su artículo 128). En este sentido, la mayoría de Estados se reservan emitir licencias obligatorias para patentes en varios supuestos, entre los que destacan dos: por necesidades de abastecimiento nacional o porque los productos farmacéuticos patentados se destinen a la exportación a países con problemas de salud pública. Este último supuesto se aplica en la Unión Europea en virtud del reglamento número 816/2006, del 17 mayo de 2006.

Según comentan los juristas, el desafío central que encierra la Directiva Europea sobre exportación es que resulte dificultoso justificar la necesidad o conveniencia de las licencias obligatorias de tecnologías que traten la COVID-19 cuando no existen problemas de abastecimiento de vacunas en el territorio de 
la Unión; y cuando, antes bien, se han comprado más vacunas de las que finalmente se han administrado. En cualquier caso, es de vital importancia tener en consideración que las posibles indemnizaciones por las licencias obligatorias quedan, en muchos países, al arbitrio de los tribunales de justicia, los cuales no aseguran remuneraciones a los titulares que sean asequibles para los Estados (Matheson y Kirkinis, 2021: 11)

Sea como fuere, el coste de exportar estas vacunas a terceros países se acerca a la mitad del precio total del tratamiento en los países más desarrollados (WHO, n.d.-c). Además, el know-how es, como ya se ha indicado, un conocimiento relevante para la fabricación y logística de las vacunas que, en tanto que no constituye una patente, sino más bien una estrategia de secreto industrial, no puede tampoco ser concedido como una licencia obligatoria. Tanta es la importancia del know-how que la propia industria farmacéutica ha tenido que cerrar acuerdos multilaterales en materia de transferencia de conocimiento, como el acuerdo entre Pfizer y BioNTech o entre Sanofi y GlaxoSmithKline.

Pero la fabricación en los países de destino de estas vacunas es un desafío todavía mayor, sobre todo porque los países en vías de desarrollo no disponen de la infraestructura necesaria ni del capital humano -no todos, está el Serum Institute of India (cf. Aars, Clarck y Schwalbe, 2021), aunque sin la distribución a gran escala todos los esfuerzos por fabricarla resultan infructuosos.

\section{Conclusiones}

Habida cuenta de la limitación de la patente como difusora de la información tecnológica que es necesaria para fabricar vacunas, es imprescindible contar con las compañías farmacéuticas en lo que respecta al know-how, los métodos de negocio, y otras modalidades de protección intelectual. El knowhow es especialmente importante en las vacunas de segunda generación, precisamente por la novedad de sus tecnologías. Aquí los métodos de fabricación "no son patógenos y, por tanto, flexibles y potencialmente exitosos a gran escala" (Aars, Clark y Schwalbe, 2021: 5). Pero, a la vez, son precisamente las compañías que tienen aprobadas vacunas de ARNm las más reacias a compartir el know-how (el caso de Moderna y la petición de los congresistas y senadores de EEUU da buena prueba de ello).

Los Estados desde luego pueden y deben emplear todas las herramientas que estén a su alcance para hacer partícipes a las empresas en esos acuerdos, pero a la vez remunerar de manera justa las inversiones de aquellas. Empero, sin olvidar qué parte de esa investigación se ha financiado con fondos públicos. También las fundaciones privadas, las ONGs y demás organizaciones filántropas, como ya hace la DCVMN, deben poder colaborar en lo posible, puesto que su acreditada experiencia en países subdesarrollados en lo que respecta a la producción y logística de vacunas y medicamentos supera en ocasiones a la de los propios Estados. Pensamos que debe exigírseles su parte en la solución de todos estos desafíos, y la sociedad ha de ser consciente de su potencia.

El desafío al que nos enfrentamos, la gestión pública de la COVID-19, es de naturaleza global. La resolución de un problema de estas características, que potencialmente afecta a todos los seres humanos, es oportuno abordarlo desde la perspectiva de la cooperación. Incluso desde una perspectiva egoísta, los países desarrollados están obligados a colaborar con el resto de nacionales del mundo si no quieren resultar dañados otra vez por las nuevas variantes y mutaciones, que a cada paso reducen la efectividad de las vacunas. Además, la importancia de la respuesta política al problema de vacunas, patentes y COVID-19 sienta un precedente sobre cómo nuestras sociedades, altamente tecnificadas, tratarán los desafíos sanitarios futuros (Krishtel y Hassan, 2021).

\section{Referencias}

Aars, O. K., Clark, M., \& Schwalbe, N. (2021). Increasing efficiency in vaccine Production: A primer for change. Vaccine: X. 8. 100104. https://doi.org/10.1016/J.JVACX.2021.100104 
Agencia Española del Medicamento. (2020, 28 julio). Recomendaciones para el tratamiento con remdesivir de pacientes con COVID-19. Recuperado 27 de octubre de 2021, de https://www.aemps.gob.es/informa/notasinformativas/medicamentosusohumano-3/2020medicamentosusohumano-3/recomendaciones-para-el-tratamiento-con-remdesivir-de-pacientes-con$\underline{\text { covid-19/ }}$

Asundi, A., O'Leary, C., \& Bhadelia, N. (2021). Global COVID-19 vaccine inequity: The scope, the impact, and the challenges. Cell Host \& Microbe. 29(7). 1036. https://doi.org/10.1016/J.CHOM.2021.06.007

Biddle, J. (2014). "Can Patents Prohibit Research? On the Social Epistemology of Patenting and Licensing in Science." Studies in History and Philosophy of Science 45. 14-23.

Cebrián, M. y López, S. (2017). “Assessing the Impact of Field-of-Use Restrictions in Patent Licensing Agreements: The Ethical Pharmaceutical Industry in the United States." 1950-1962. Enterprise \& Society. 18(2). 282-323.

Chandrasekharan, S., Amin, T., Kim, J., Furrer, E., Matterson, A. C., Schwalbe, N., \& Nguyen, A. (2015). "Intellectual property rights and challenges for development of affordable human papillomavirus, rotavirus and pneumococcal vaccines: Patent landscaping and perspectives of developing country vaccine manufacturers.” Vaccine. 33(46). 6366-6370.

Czochor, J., \& Turchick, A. (2014). Introduction. The Yale Journal of Biology and Medicine. 87(4). 401. Retrieved from /pmc/articles/PMC4257027/

Denicolò, V., \& Franzoni, L. A. (2003). “The contract theory of patents.” International Review of Law and Economics. 23(4). 365-380. https://doi.org/10.1016/J.IRLE.2003.07.002

Domínguez, N. (2021, 20 mayo). "Suspender las patentes no resolverá la escasez de vacunas". El País. Recuperado de https://elpais.com

Dong, Y., Dai, T., Wei, Y., Zhang, L., Zheng, M., \& Zhou, F. (2020). “A systematic review of SARSCoV-2 vaccine candidates". Signal Transduction and Targeted Therapy. 5(1). https://doi.org/10.1038/S41392-020-00352-Y

Dutfield, G. (2003). Intellectual Property Rights and the Life Science Industries A Twentieth Century History. Routledge. Abingdon, Reino Unido.

Eccleston-Turner, M. (2017). "Beyond patents: Scientific knowledge, and access to vaccine." Ethics, Medicine and Public Health. 3(1). 64-73. https://doi.org/10.1016/J.JEMEP.2017.02.011

Eccleston-Turner, Mark, \& Upton, H. (2021). "International Collaboration to Ensure Equitable Access to Vaccines for COVID-19: The ACT-Accelerator and the COVAX Facility". The Milbank Quarterly. 99(2). 426. https://doi.org/10.1111/1468-0009.12503

Forman, R., Shah, S., Jeurissen: , Jit, M., \& Mossialos, E. (2021). "COVID-19 vaccine challenges: What have we learned so far and what remains to be done?" Health Policy. 125(5). 553-567. https://doi.org/10.1016/J.HEALTHPOL.2021.03.013

Gaviria, M., \& Kilic, B. (2021). A network analysis of COVID-19 mRNA vaccine patents. Nature Biotechnology 2021 39. 5. 39(5). 546-548. https://doi.org/10.1038/s41587-021-00912-9

Health Action International. (n.d.). C-TAP has not (yet) lived up to high expectations - Health Action International. Retrieved from https://haiweb.org/c-tap-has-not-yet-lived-up-to-high-expectations/ 
Henry, B. (2018). "Drug pricing and challenges to hepatitis C treatment access." Journal of health \& biomedical law. 14(0). 265-283.

Krishtel: , \& Hassan, F. (2021). "Share vaccine know-how." Science. 374(6566). 379-379. https://doi.org/10.1126/SCIENCE.ABM8724

Martínez, S. (2021, 10 junio). "La Eurocámara reclama liberar las patentes de las vacunas contra el covid”. El Periódico. Recuperado de https://www.elperiodico.com

Mateos, A. (2021, 10 junio). "Sí del Parlamento Europeo a eliminar patentes en vacunas Covid". Redacción Médica. Recuperado de https://www.redaccionmedica.com

Matheson Am, S., \& Kirkinis, A. (2021). "Compulsory licence and Crown use provisions in the Covid19 pandemic - the Australian perspective”. Journal of Intellectual Property Law \& Practice. 16(6). 484 497. https://doi.org/10.1093/JIPLP/JPAB070

McAdams, D., McDade, K. K., Ogbuoji, O., Johnson, M., Dixit, S., \& Yamey, G. (2020). “Incentivising wealthy nations to participate in the COVID-19 Vaccine Global Access Facility (COVAX): a game theory perspective". BMJ Global Health. 5(11). https://doi.org/10.1136/BMJGH-2020-003627

McMahon, A. (2020). "Global equitable access to vaccines, medicines and diagnostics for COVID-19: The role of patents as private governance". Journal of Medical Ethics. 47(3). 142-148. https://doi.org/10.1136/MEDETHICS-2020-106795

Moon, S., Alonso Ruiz, A., \& Vieira, M. (2021). "Averting Future Vaccine Injustice. New England" Journal of Medicine. 193 385(3). https://doi.org/10.1056/NEJMP2107528/SUPPL FILE/NEJMP2107528 DISCLOSURES.PDF

Médicos Sin Fronteras. (2021, 7 abril). Pedro Sánchez: no a las patentes, la pandemia no es un negocio. Recuperado 19 de octubre de 2021, de https://www.msf.es/firma-patente-vacuna-covid

Nordhaus, W. D. (1969). "An Economic Theory of Technological Change”. The American Economic Review. 59(2). 18-28. http://www.jstor.org/stable/1823649

Parthasarathy, S. (2017). Patent Politics Life Forms, Markets, and the Public Interest in the United States and Europe. University of Chicago Press. Chicago, EEUU.

Peacocke, E. F., Heupink, L. F., Frønsdal, K., Dahl, E. H., \& Chola, L. (2021). “Original research: Global access to COVID-19 vaccines: a scoping review of factors that may influence equitable access for low and middle-income countries". BMJ Open. 11(9). https://doi.org/10.1136/BMJOPEN-2021-049505

Perehudoff, K., Thoen, E., \& Boulet: (2021). "Overriding drug and medical technology patents for pandemic recovery: a legitimate move for high-income countries, too." BMJ Global Health. 6(4). e005518. https://doi.org/10.1136/BMJGH-2021-005518

Sánchez, V. (2021, 3 junio). "Covid-19: ¿por qué hay oposición al levantamiento de patentes de las vacunas?" France24. Recuperado de https://www.france24.com

The Guardian. (n.d.). "WHO platform for pharmaceutical firms unused since pandemic began | World Health Organization" The Guardian. Retrieved from https://www.theguardian.com/world/2021/jan/22/who-platform-for-pharmaceutical-firms-unusedsince-pandemic-began

The Pharma Letter. (2021, 1 junio). Russian government finally approves compulsory licensing of original drugs. Recuperado 2 de noviembre de 2021, de 
https://www.thepharmaletter.com/article/russian-government-finally-approves-compulsory-licensingof-original-drugs

Uddin, M., Mustafa, F., Rizvi, T. A., Loney, T., Al Suwaidi, H., Al-Marzouqi, A. H. H., ... Senok, A. C. (2020). "SARS-CoV-2/COVID-19: Viral Genomics, Epidemiology. Vaccines, and Therapeutic Interventions." Viruses. 12(5). https://doi.org/10.3390/V12050526

Usher, A. D. (2020). "South Africa and India push for COVID-19 patents ban". The Lancet. 396(10265). 1790-1791. https://doi.org/10.1016/S0140-6736(20)32581-2

van de Pas, R., Widdowson, M.-A., Ravinetto, R., N Srinivas: , Ochoa, T. J., Fofana, T. O., \& Van Damme, W. (2021). "COVID-19 vaccine equity: a health systems and policy perspective". Expert Review of Vaccines. https://doi.org/10.1080/14760584.2022.2004125

van Riel, D., \& de Wit, E. (2020). “Next-generation vaccine platforms for COVID-19”. Nature Materials 2020 19:8. 19(8). 810-812. https://doi.org/10.1038/s41563-020-0746-0

Wang, J., Peng, Y., Xu, H., Cui, Z., \& Williams, R. O. (2020). "The COVID-19 Vaccine Race: Challenges and Opportunities in Vaccine Formulation". AAPS PharmSciTech. 21(6). https://doi.org/10.1208/S12249-020-01744-7

Warren, E. (2021, 12 de octubre). Carta a WH y BARDA sobre el Contrato de Moderna https://www.warren.senate.gov/imo/media/doc/2021.10.12\%20Letter\%20to\%20WH\%20and\%20BAR DA\%20on\%20Moderna\%20Contract.pdf

WHO. (n.d.-a). C-TAP: A concept paper. Retrieved November 15, 2021, from https://www.who.int/publications/m/item/c-tap-a-concept-paper

WHO. (n.d.-b). COVID-19 technology access pool. Retrieved November 15, 2021, from https://www.who.int/initiatives/covid-19-technology-access-pool

WHO. (n.d.-c). "Costs of Delivering COVID-19 Vaccine in 92 AMC Countries: Updated Estimates from COVAX Working Group on Delivery Costs." Retrieved November 15. 2021 from https://www.who.int/docs/default-source/coronaviruse/act-accelerator/covax/costs-of-covid-19vaccine-delivery-in-92amc_08.02.21.pdf

WIPO. (2012). Patent Landscape Report on Vaccines for Selected Infectious Diseases. Recuperado de https://www.wipo.int/edocs/pubdocs/en/patents/946/wipo_pub_946_3.pdf

WTO. (n.d.). WTO | intellectual property (TRIPS) - agreement text - standards. Retrieved November 15, 2021, from https://www.wto.org/english/docs e/legal e/27-trips 04c e.htmgestión 